\title{
ARTICLE OPEN \\ Reversible and nonvolatile manipulation of the electronic transport properties of topological insulators by ferroelectric polarization switching
}

Xu-Wen Zhao', Si-Ning Dong ${ }^{2,3}$, Guan-Yin Gao ${ }^{4}$, Zhi-Xue Xu' ${ }^{1}$, Meng Xu' ${ }^{1}$, Jian-Min Yan ${ }^{1}$, Wei-Yao Zhao ${ }^{1}$, Yu-Kuai Liu ${ }^{5}$, Shu-Ying Yan ${ }^{6}$, Jin-Xing Zhang ${ }^{6}$, Yu Wang ${ }^{7}$, Hai-Zhou Lu ${ }^{8}{ }^{8}$, Xiao-Guang Li ${ }^{4}{ }^{4}$, J. K. Furdyna ${ }^{2}$, Hao-Su Luo ${ }^{1}$ and Ren-Kui Zheng ${ }^{1,7}$

Reversible and nonvolatile electric-field control of the physical properties of topological insulators is essential for fundamental research and development of practical electronic devices. Here, we report the integration of topological insulator films with ferroelectric $\mathrm{Pb}\left(\mathrm{Mg}_{1 / 3} \mathrm{Nb}_{2 / 3}\right)_{3}-\mathrm{PbTiO}_{3}$ (PMN-PT) single crystals in the form of ferroelectric field-effect devices that allow us to tune the electronic properties of topological insulator films in a reversible and nonvolatile manner. Specifically, gating of $\mathrm{Cr}_{-}-\mathrm{doped}_{\mathrm{Bi}} \mathrm{Se}_{3}$ films with the PMN-PT layer is shown to provide a means to reversibly tune and modulate the carrier density and carrier type, as well as its other properties, such as the conductance, magnetoconductance, Fermi level, phase coherence length, and screening factor of electron-electron interaction by polarization switching at room temperature. These findings provide a simple and direct approach for probing the quantum transport properties of topological insulator films through ferroelectric gating by using PMN-PT. The combination of topological insulators with both ferroelectrically and piezoelectrically active PMN-PT thus offers a promising step toward exploring topological insulator/ferroelectric(piezoelectric) hybrid devices that could utilize not only the ferroelectric field-effect of topological insulator/PMN-PT structures but also the unique properties of respective materials.

npj Quantum Materials (2018)3:52 ; doi:10.1038/s41535-018-0125-0

\section{INTRODUCTION}

Topological insulators (TIs) are a new class of quantum materials that continue to attract worldwide attention due to a variety of novel phenomena that they support, such as the topological magnetoelectric effect, Majorana fermions, quantized anomalous Hall effect, topological superconductor, and their potential applications in quantum computation and dissipationless spintronic devices. ${ }^{1-10}$ A number of experimental techniques, including chemical doping, ${ }^{3-5}$ thickness variation, ${ }^{6,7}$ mechanical and epitaxial strain, ${ }^{8-12}$ hydrostatic pressure, ${ }^{13}$ magnetic proximity, ${ }^{14,15}$ and electric gating, ${ }^{7,16-25}$ have been employed in studies of quantum transport properties of Tls. Among these techniques, electric gating offers an effective approach for tailoring the electronic properties of Tls since it enables in situ modification of carrier density and carrier type and thus circumvents a number of problems inherent to TI films, e.g., disorders, ${ }^{12,26}$ defects, $^{12,27}$ and lattice strains. ${ }^{8-12}$

Several dielectric insulators such as the $\mathrm{SiO}_{2},{ }^{3,28} \mathrm{Al}_{2} \mathrm{O}_{3},{ }^{16} \mathrm{HfO}_{2}{ }^{17}$ $h$-BN, ${ }^{18} \mathrm{SiNx}{ }^{19}$ and $\mathrm{SrTiO}_{3}{ }^{20-22}$ have been used as gate materials to tune the carrier density and carrier type, Fermi level, and electronic transport properties of TI films through the application of gate voltages at very low temperatures (millikelvin to several kelvin). It is noted that the electric-field-induced polarization charges achieved by gating with these dielectric insulators used so far for this purpose are itself volatile and involve relatively weak areal charge density $\left(\sim 10^{12}-10^{13} / \mathrm{cm}^{2}\right),{ }^{3,16-22,28}$ making it impossible to manipulate the carrier density and carrier type of TI films in a nonvolatile manner at ambient temperatures. Although ionic liquid or gel-like electrolyte gate materials have been successfully used to tune the carrier density in a variety of thin-film samples, including topological insulators, ${ }^{25}$ iron-based superconductors, ${ }^{29}$ two-dimensional (2D) chalcogenides, ${ }^{30}$ tungsten oxides, ${ }^{31}$ wideband-gap oxide semiconductors, ${ }^{32}$ and strongly correlated oxide magnetics, ${ }^{33}$ these electrolytes are not compatible with modern solid-state electronics and may react with thin-film samples electrochemically when gate voltages are applied. 25,29-31

To make progress in both basic research and practical applications, it is desirable to realize control of both carrier density and carrier type and related electronic properties of $\mathrm{TI}$ films in a reversible and nonvolatile manner at room temperature. Electrostatic manipulation of carrier density via ferroelectric gating provides a promising approach for realizing such goals. Among a number of ferroelectric materials, perovskite $\mathrm{Pb}\left(\mathrm{Mg}_{1 / 3} \mathrm{Nb}_{2 / 3}\right)$ $\mathrm{O}_{3}-\mathrm{PbTiO}_{3}$ (PMN-PT) single crystals possess ultra-high piezoelectric coefficients $\left(d_{33}>2000 \mathrm{pC} / \mathrm{N}\right)$ and large remnant polarization of $2 \mathrm{Pr} \sim 60-80 \mu \mathrm{C} / \mathrm{cm}^{2}$ at room temperature, which

\footnotetext{
${ }^{1}$ State Key Laboratory of High Performance Ceramics and Superfine Microstructure, Shanghai Institute of Ceramics, Chinese Academy of Sciences, Shanghai 200050, China; ${ }^{2}$ Department of Physics, University of Notre Dame, South Bend, IN 46556, USA; ${ }^{3}$ Materials Science Division, Argonne National Laboratory, Argonne, IL 60439, USA; ${ }^{4}$ Hefei National Laboratory for Physical Sciences at Microscale and Department of Physics, University of Science and Technology of China, Hefei 230026 , China; ${ }^{5}$ Department of Applied Physics, The Hong Kong Polytechnic University, Hong Kong, China; ${ }^{6}$ Department of Physics, Beijing Normal University, Beijing 100875, China; ${ }^{7}$ School of Materials Science and

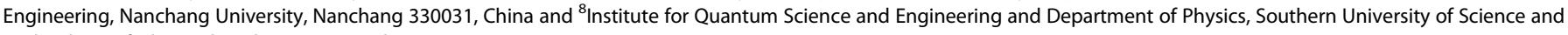
Technology of China, Shenzhen 518055, China

Correspondence: Y-K. Liu (liuyk@mail.ustc.edu.cn) or Yu Wang (wangyu@ncu.edu.cn) or R-K. Zheng (zrk@ustc.edu)

These authors contributed equally: Xu-Wen Zhao, Si-Ning Dong
}

Received: 29 April 2018 Revised: 30 September 2018 Accepted: 3 October 2018

Published online: 19 October 2018 

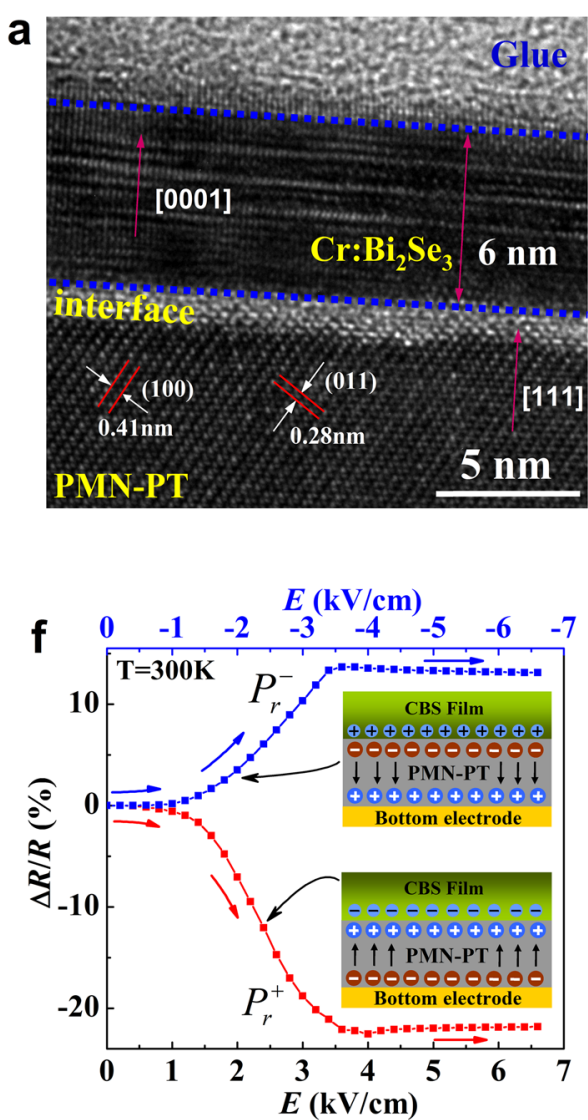
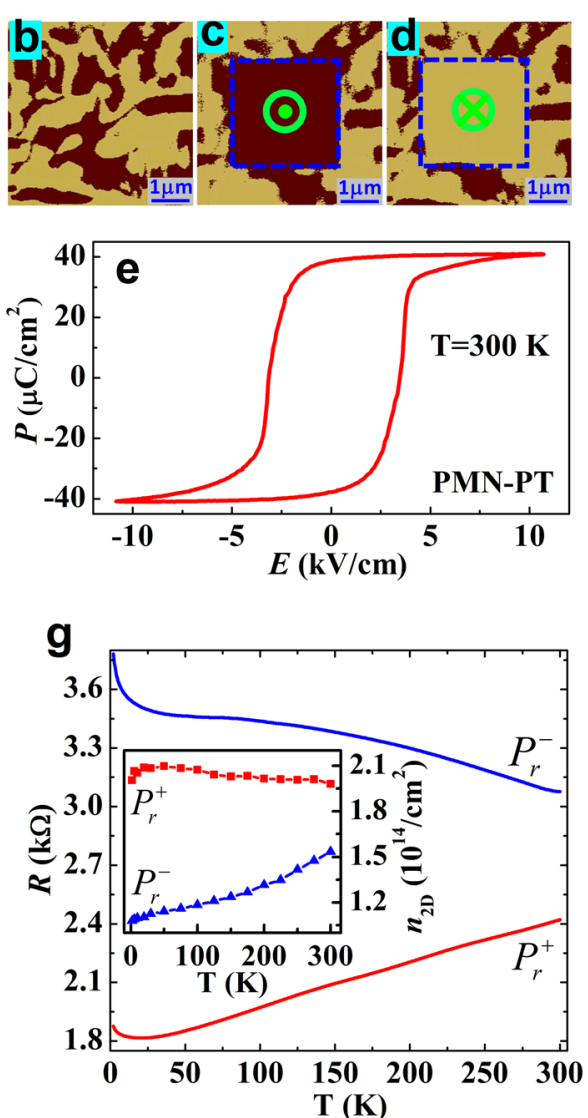

Fig. 1 Structural, ferroelectric, piezoelectric, and electronic properties of the CBS(6 nm)/PMN-PT structure. a Cross-sectional TEM image. b-d Out-of-plane PFM images of different ferroelectric domain states of the PMN-PT substrate. e Polarization-electric-field hysteresis loop of the PMN-PT substrate. $f$ Relative resistance change $(\Delta R / R)$ of the CBS film obtained by scanning the electric fields from zero to positive (red) and zero to negative (blue). Before scanning the electric fields the PMN-PT substrates were in the unpoled state. Insets: Schematic illustrations for the positively poled $P_{r}^{+}$and negatively poled $P_{r}^{-}$states of the PMN-PT. $\mathbf{g}$ Temperature dependence of resistance of the CBS film for the $P_{r}^{+}$and $P_{r}^{-}$states of the PMN-PT. Inset: Areal carrier density $n_{2 D}$ as a function of temperature for the two polarization states of the PMN-PT

corresponds to an areal charge density of $\sim 3.7-4.9 \times 10^{14} / \mathrm{cm}^{2}$. This value is one order of magnitude higher than that obtainable with the dielectric gate materials mentioned above (Supplementary SI). For bismuth chalcogenide TI films, the areal carrier density $\left(n_{2 D}\right)$ is generally on the order of $\sim 10^{13} / \mathrm{cm}^{23,6,7,16,22,25,34}$, which is approximately one order of magnitude lower than the remnant polarization charges of PMN-PT. It is therefore anticipated that the Fermi level, carrier density and carrier type, and electronic properties of TI films could be significantly modulated by the nonvolatile polarization charges of PMN-PT.

For this purpose, we have fabricated TI-based ferroelectric fieldeffect transistors (FeFETs) by growing $\mathrm{Cr}$-doped $\mathrm{Bi}_{2} \mathrm{Se}_{3}$ (CBS) thin films with different thicknesses on (111)-oriented PMN-PT singlecrystal substrates. Using these TI/PMN-PT structures, we are able to successfully realize reversible and nonvolatile electric-fieldcontrol of the Fermi level, carrier density, and corresponding electronic properties of CBS films through polarization switching of PMN-PT at room temperature, thus leading to a better understanding of carrier-density-related quantum transport properties of TI films on the one hand, and providing a straightforward approach to realize reversible and nonvolatile tuning of the Fermi level and electronic properties of $\mathrm{TI}$ films on the other, as discussed in the sections that follow.

\section{RESULTS AND DISCUSSION}

Figure $1 \mathrm{f}$ shows the relative resistance changes $(\Delta R / R)$ of the $6-\mathrm{nm}$ CBS film (Fig. 1a) obtained by scanning electric fields from zero to positive (red) and zero to negative (blue), respectively. Note that the PMN-PT substrates were in the unpoled state before scanning the electric fields. The results reveal that both the magnitude and the sign of $\Delta R / R$ strongly depend on the polarization direction of the PMN-PT. $\triangle R / R$ totally decreases by $\sim 22 \%$ upon positive polarization. In contrast, $\Delta R / R$ increases by $\sim 13 \%$ upon negative polarization. We further measured the resistance of the 6-nm CBS film by scanning bipolar electric fields and observed a resistanceelectric-field hysteresis loop (Supplementary Figure S3), which implies that the interfacial charge effect plays a dominant role in controlling the resistance changes. This feature, together with the opposite changes in the sign of $\Delta R / R$ upon positive and negative polarization, demonstrates that the lattice strain effect due to the electrostriction is very weak. Further, we found that with increasing film thickness from 3 to $80 \mathrm{~nm}, \Delta R / R$ decreases significantly (Supplementary Figure S4A). Particularly, for the thickest $80-\mathrm{nm}$ film, the polarization switching has a minor effect on the resistance $(\Delta R / R=5 \%$ at $300 \mathrm{~K})$. Taking these polarization direction and thickness-dependent behaviors of $\Delta R / R$ and the $n$ type nature of charge carriers of the CBS films (Supplementary Figure S4D) into account, we conclude that the electric-fieldinduced positive or negative polarization charges at the interface contribute significantly to the resistance changes. We thus measured the carrier density of the CBS films by Hall measurements using the Van der Pauw configuration (Supplementary Figure S2A). The areal carrier density $n_{2 D}$ for the positively polarized $P_{r}^{+}$and negatively polarized $P_{r}^{-}$states is plotted as a function of temperature in the inset of Fig. $1 \mathrm{~g}$. Indeed, $n$ was 

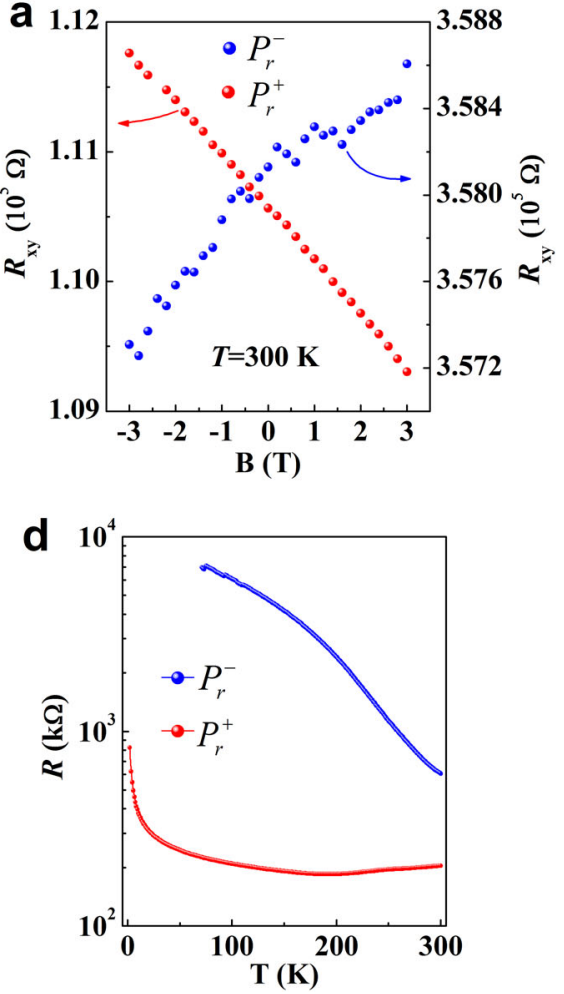

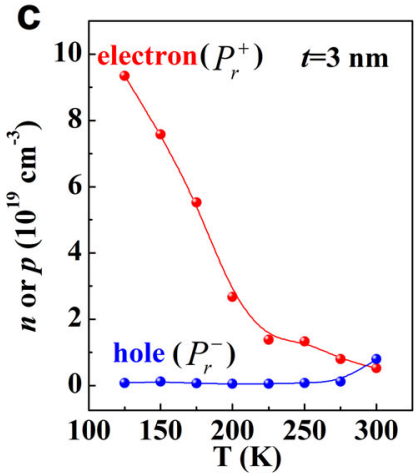

e

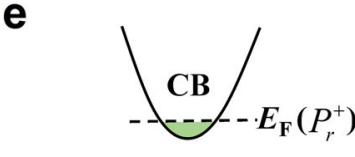

\section{Reversible and}

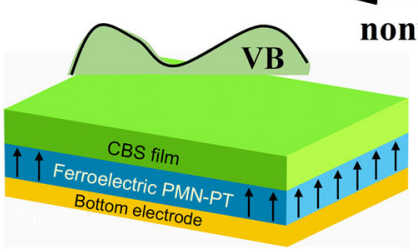

f
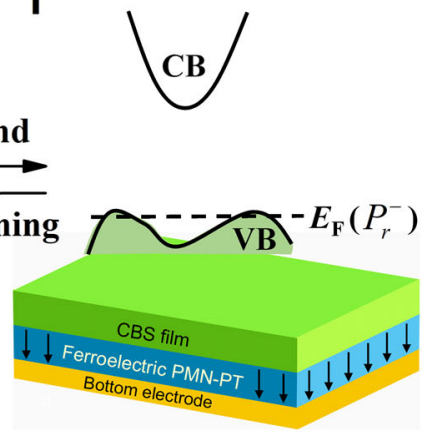

Fig. 2 Hall resistance, carrier density, and resistance of the $\sim 3 \mathrm{~nm}$ CBS film. $\mathbf{a}, \mathbf{b}$ Hall resistance as a function of the magnetic field at $T=300$ and $200 \mathrm{~K}$ for the $P_{r}^{+}$and $P_{r}^{-}$states of the PMN-PT substrate. $\mathbf{c}, \mathbf{d}$ Temperature dependence of the charge-carrier density and resistance of the CBS film for the $P_{r}^{+}$and $P_{r}^{-}$states of the PMN-PT substrate. e, $\mathbf{f}$ Schematic band diagrams of the Fermi level shift induced by polarization switching

modified by $\sim 46 \%$ at $2 \mathrm{~K}$ and by $\sim 23 \%$ at $300 \mathrm{~K}$ as the polarization is switched from $P_{r}^{+}$to $P_{r}^{-}$, while the electron mobility $\mu$ is only weakly modified by the polarization switching (Supplementary Figure S4B). For the present PMN-PT with a rhombohedral crystal structure the polarization switching from $P_{r}^{+}$(i.e., the [111] direction) to $P_{r}^{-}$(i.e., the [-1-1-1] direction), as schematically illustrated in Figure S5 (Supplementary Materials), should not induce any lattice strain, ${ }^{35,36}$ which is confirmed by the X-ray diffraction results shown in Figure S6 (Supplementary Materials), where the PMN-PT(111) and CBS(0003), (0006), (00015) diffraction peaks remain unchanged as the polarization state was switched from $P_{r}^{+}$to $P_{r}^{-}$. Further, PFM measurements show that the polarization direction had been fully and reversibly switched between the [111] and the [-1-1-1] directions by electric voltages, as evidenced by the uniform and sharp contrast between the two domain states within the dotted blue boxes (Fig. 1c, d). These XRD and PFM results demonstrate that any possible lattice strain effects associated with the polarization switching can not account for the resistance and carrier density differences between the two polarization states of PMN-PT. Our findings thus strongly suggest that the polarization switching-induced interfacial charge effects play a crucial role in estalishing the electronic changes of the CBS/ PMN-PT structure.

Due to the nonvolatile nature of polarization charges at the interface, ${ }^{35,36}$ the resistance for the $P_{r}^{+}$and $P_{r}^{-}$states exhibits distinct differences down to the lowest temperature for all CBS films with different thicknesses (Fig. $1 \mathrm{~g}$ and Supplementary Figure $\mathrm{S} 4 \mathrm{~A})$. As can be seen in Fig. $1 \mathrm{~g}$, for the $P_{r}^{+}$state the resistance shows metallic conducitivity $(d R / d T>0)$ for $T>20 \mathrm{~K}$ and a weak increase for $T \leq 20 \mathrm{~K}$. Upon polarization switching from $P_{r}^{+}$to $P_{r}^{-}$, the metallic profile of the resistance is converted to a semiconducting one $(d R / d T<0)$, with a stronger resistance upturn for $T \leq 20 \mathrm{~K}$, which can be understood by the significant lowering of the areal carrier density $\left(n_{2 D}\right)$ by amount of $\Delta n_{2 D} \sim 7.7 \times 10^{13}$ / $\mathrm{cm}^{2}$ at $T=2 \mathrm{~K}$ and $\Delta n_{2 D} \sim 3.7 \times 10^{13} / \mathrm{cm}^{2}$ at $T=300 \mathrm{~K}$ (inset, Fig. $1 \mathrm{~g})$. Such a large reduction in $n_{2 D}$ would result in lowering of the Fermi level relative to the valence band, ${ }^{1,37}$ thus leading to a metal-to-semiconductor transition. Moreover, we found that the polarization switching between $P_{r}^{+}$and $P_{r}^{-}$results not only in the changes in the carrier density (Fig. 2c) and resistance (Fig. $2 d$ ), but also in a reversible and nonvolatile switching of the carrier type between $n$-type and $p$-type in the thinnest CBS film $(\sim 3 \mathrm{~nm})$, as evidenced by the reversible changes in the sign of the slope of the Hall resistance $R_{x y}$ versus the magnetic field $B$ curves at $T=300$ and $200 \mathrm{~K}$ (Fig. 2a, b). For such a thin CBS film, the top surface state and the bottom surface state would hybridize, resulting in a hybridized gap between the conduction band and the valence band. Thus, the Hall results imply that the Fermi level could be reversibly tuned between the conduction band and the valence band by the polarization switching of the PMN-PT layer at room temperature, as schematically illustrated by Fig. 2e, f. Although such reversible switching of carrier type between $n$ and $p$ has been achieved earlier, in earlier works this has only been successful at extremely low temperatures and only in a volatile manner ${ }^{16-22}$ or has been achieved in a irreversible manner via chemical doping. ${ }^{4,5,28,38,39}$ Our approach of PMN-PT-based FeFET structure has the advantage of enabling in situ reversible and nonvolatile tuning of carrier density and carrier type, achievable at room temperature. As seen in Fig. 1a, the quality of our 6-nm CBS film prepared by the pulse laser deposition is indeed lower than those prepared by the molecular beam expitaxy (MBE). ${ }^{6,10,14,22,26,34,37}$ As a result, the areal carrier density of the 6-nm CBS film $\left(n_{2 D} \sim 1.8 \times 10^{14} / \mathrm{cm}^{2}\right)$ is approximately one order of magnitude higher that those prepared by MBE. ${ }^{6,22,34,37}$ Consequently, the polarization direction switching of the PMN-PT layer is unable to convert the carrier type of the $6-\mathrm{nm}$ film from $n$-type to 

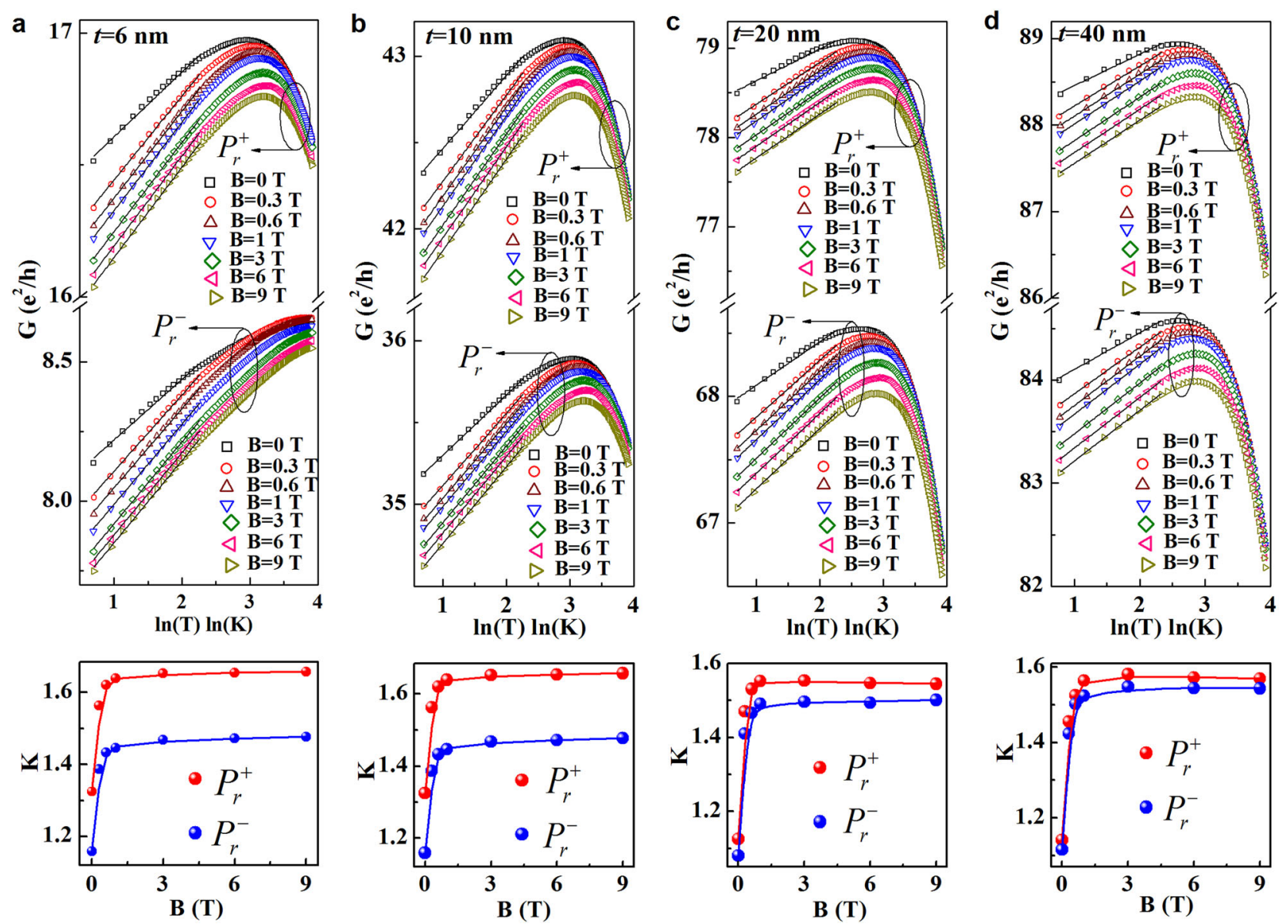

Fig. 3 Logarithmic temperature dependence of conductance $G\left(e^{2} / h\right)$ in different magnetic fields measured for CBS films with different thicknesses when the PMN-PT substrates are in the $P_{r}^{+}$and $P_{r}^{-}$states, respectively. The black solid lines are linear fitting results obtained with Eq. (1). Lower panels of a-d show the magnetic field dependence of the coefficient $\mathrm{K}$ extracted from Eq. (1) for both polarization states of PMN-PT, respectively

p-type. Nevertheless, it is anticipated that if high-quality TI films with lower carrier density is prepared on PMN-PT using MBE, the carrier type of thicker CBS films $(t>3 \mathrm{~nm}$ ) would also be reversibly modulated between $n$ and $p$ in a nonvolatile manner by polarization switching. In any case, the present realization of reversible modulation of carrier type in a nonvolatile manner by polarization switching at room temperature is an encouraging outcome for both basic research and practical applications and demonstrate the feasibility of carrier type modulation using ferroelectric gating at ambient temperature. Finally, it is noted that regardless of the polarization states of PMN-PT, the loffe-Regel parameters $k_{F} \ell^{40}$ for all films are larger than unity (Supplementary Figure S4C), indicating that the electronic transport in this system is in the weakly disordered regime. $22,26,41$

We note that the resistance of all CBS films shows upturn at low temperatures with decreasing temperature $(d R / d T<0)$ and are enhanced by the application of magnetic fields for both $P_{r}^{+}$and $P_{r}^{-}$ states (Supplementary Figure S7). Magnetic measurements indicate that the doped $\mathrm{Cr}$ ions did not form long-range ferromagnetic order (Supplementary Figure S8), which is similar to that observed in MBE-grown CBS films with high- $\mathrm{Cr}$ doping levels $(\geq 9.9 \%) .{ }^{42,43}$ Spin-dependent magnetic scattering is clearly unable to account for the enhancement of the resistance in magnetic fields. Nevertheless, these temperature- and magnetic field-dependent resistance behaviors may be reasonably understood by taking both the electron-electron interaction (EEI) and quantum interference into account. ${ }^{22,41,44,45}$ To get insight into this, we analyzed the low-temperature resistance behaviors in terms of the 2D Dirac model, which takes both EEl and quantum interference into account. ${ }^{41}$ To perform the fitting, we present the resistance data (Supplementary Figure S7) in the form of quantized conductance $G\left(e^{2} / h\right)$, where $e$ and $h$ are the unit charge and Planck constant, respectively. Figure $3 a, b, c, d$ show the logarithmic temperature dependence of $G\left(e^{2} / h\right)$ for the CBS films with different thicknesses $(6 \mathrm{~nm} \leq t \leq 40 \mathrm{~nm})$ in magnetic fields up to $9 \mathrm{~T}$ when the adjacent PMN-PT layers were in the $P_{r}^{+}$and $P_{r}^{-}$ states, respectively. In all cases, $G\left(e^{2} / h\right)$ decreases linearly with decreasing temperature from $10 \mathrm{~K}$ down, and can be satisfactorily fitted in the temperature range from 2 to $10 \mathrm{~K}$ by the equation(41),

$$
G(T)=G_{0}+K\left(e^{2} / 2 \pi^{2} \hbar\right) \ln \left(T / T_{0}\right)
$$

where $K$ is the slope of the temperature-dependent conductance, which reflects the contribution of the electron-electron interaction and quantum interference to the surface state conductivity at low temperatures. $G_{0}$ is the residual conductance. $T_{0}$ is a reference temperature from which one measures the deviation $\Delta G$. The coefficient $K$ for the $P_{r}^{+}$and $P_{r}^{-}$states are plotted against the magnetic field $B$ in the lower panels of Fig. 3a, b, c, d, respectively. For both polarization states, $K$ increases sharply with increasing field within a narrow-field window below $B=1 \mathrm{~T}$, and saturates for $B>1 \mathrm{~T}$. Such a variation of $K$ with $B$ is consistent with the theoretically calculated results, which are obtained based on the corrections to conductivity from EEI and quantum interference for disordered massless Dirac fermions. This, together with the good fits of $G$ to Eq. (1) suggests the existence of surface states at low temperatures for the 6 - to $40-\mathrm{nm}$ CBS film. ${ }^{41}$ Upon polarization 

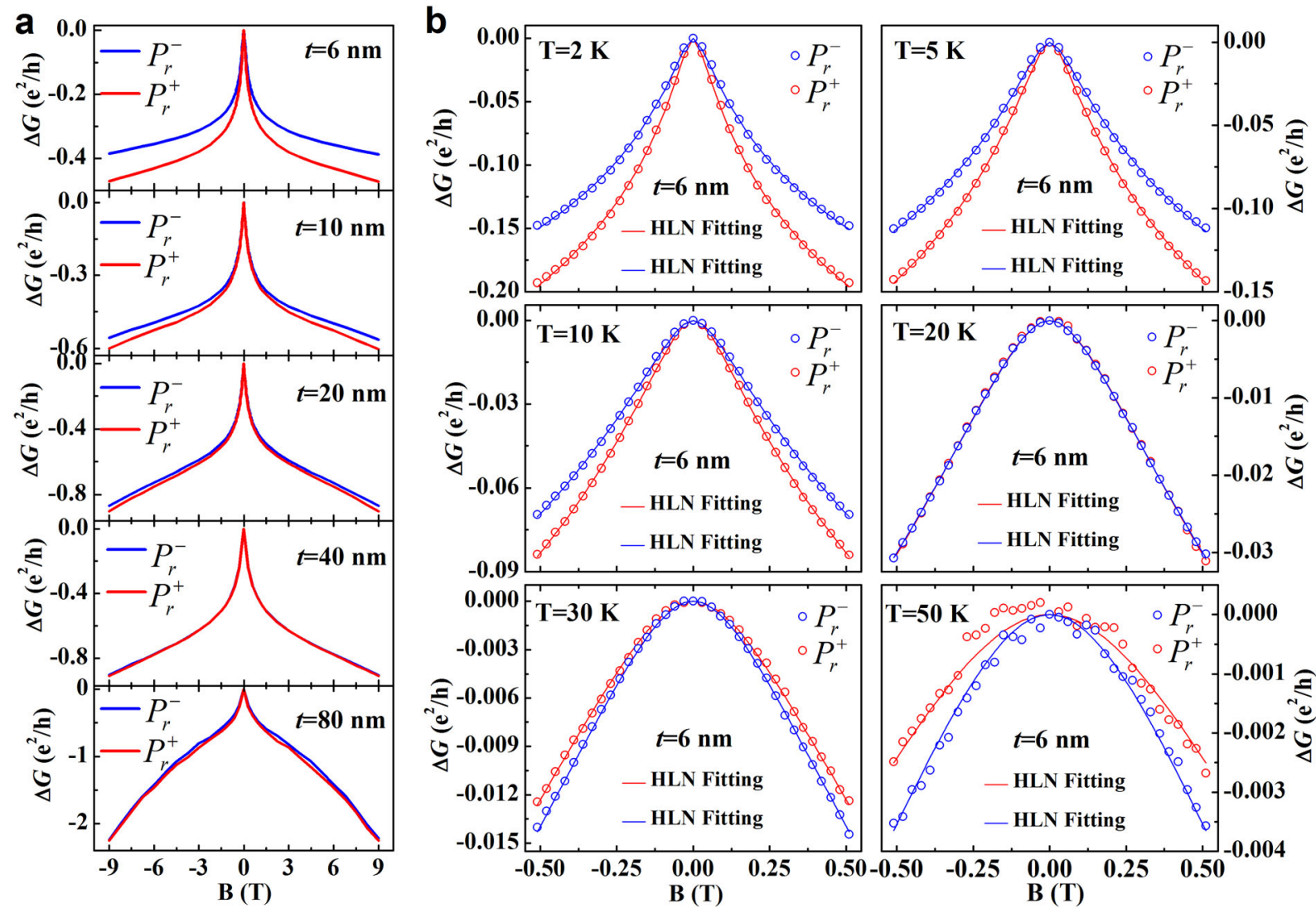

Fig. 4 Magnetoconductance $\Delta G$ versus magnetic field $B$ for CBS films for PMN-PT substrates in $P_{r}^{+}$and $P_{r}^{-}$states. a $\Delta G$ for CBS films with different thicknesses measured at a fixed temperature $(T=2 \mathrm{~K})$. b $\Delta G$ for the $6 \mathrm{~nm}$ CBS film, as measured at different temperatures of $T=2,5$, $10,20,30$, and $50 \mathrm{~K}$, respectively. The blue and red solid curves represent the fitting results obtained using Eq. (3)

switching from $P_{r}^{+}$to $P_{r}^{-}, K$ is seen to decrease appreciably, particularly for the thinner $6-$ and $10-\mathrm{nm}$ CBS films. It has been shown that $K$ can be written as $K / \pi=K^{e e}+K^{q i}$, where $K^{\mathrm{ee}} \approx 1-\left(\eta_{\wedge}\right.$ $\left.+\eta_{\Gamma}\right) F$ is a contribution from $\mathrm{EEl}$, and $K^{q i}=a p$ is a contribution from quantum interference. ${ }^{41}$ Here, $\eta_{\Lambda} \in[1 / 2,1]$ and $\eta_{\Gamma} \in[1 / 4,0]$ are weighting factors, and $F \in[0,1]$ is the screening factor of electron-electron interaction. In $K^{a i}=a p, a$ is a prefactor that describes the number of conductance channels and the parameter $p$ can be obtained from fitting of the phase coherence length $\ell_{\phi}$ to $\ell_{\phi} \sim T^{-p / 2}$ (as discussed in sections that follow). In highmagnetic fields $\left(B \gg B_{\phi}\right.$, where $B_{\phi}$ is the coherence field $B_{\phi}=\hbar / 4 \mathrm{e} \ell_{\phi}^{2}$ ) destructive quantum interference is greatly suppressed, resulting in $K^{q i} \rightarrow 0$. The constant $K$ is then solely determined by $K^{e e}$, i.e.,

$K / \pi=K^{\mathrm{ee}} \approx 1-\left(\eta_{\Lambda}+\eta_{\Gamma}\right) F$

For the 6-nm CBS film, upon switching polarization from $P_{r}^{+}$to $P_{r}^{-}$, the term $\eta_{\Lambda}+\eta_{\Gamma}$ decreases slightly, from 0.70 to 0.68 (see Supplementary Figure S9 for details). This, together with Eq. (2) indicates that the screening factor $F$ increases as a result of the polarization switching-induced reduction in the areal carrier density $n_{2 D}$. In the limit $B<<B_{\phi}$, on the other hand, both $K^{e e}$ and $K^{q i}$ contribute to $K$. One then has $K / \pi=K^{e e}+K^{q i}=1+a p-$ $\left(\eta_{\wedge}+\eta_{\Gamma}\right) F^{41}$ Since, as seen in Fig. 5 and Supplementary Figure S9A, $a$ increases as the polarization is switched from $P_{r}^{+}$to $P_{r}^{-}$, thus the decrease in $K$ implies that the screening factor $F$ increases upon the polarization switching from $P_{r}^{+}$to $P_{r}^{-}$. Namely, for $B<<$ $B_{\phi} F$ also increases as a result of the reduction in $n_{2 D}$. Therefore, the results experimentally demonstrate that the polarization switching-induced reduction in the areal carrier density enhances the screening factor of the electron-electron interaction, thereby modifying the EEI contribution, which is consistent with the theoretically calculated results from the $\mathrm{Bi}_{2} \mathrm{Se}_{3}$ and $\mathrm{Pb}$-doped $\mathrm{Bi}_{2} \mathrm{Se}_{3}$ films (ref. ${ }^{45}$ ).

Now, we turn to the effects of polarization switching on the magnetoconductance of CBS films with different thicknesses and at different fixed temperatures. It is known that topological surface states can be identified by observing quantum corrections to the conductance in the form of WAL at low temperatures, which manifests itself as a sharp cusp in magnetoconductance. $^{41,46,47}$ Figure $4 a$ shows the magnetoconductance $(\Delta G)$ as a function of B for CBS films with different thicknesses $(t=6,10$, $20,40,80 \mathrm{~nm}$ ). Negative $\Delta G$ with a sharp cusp at $B=0 \mathrm{~T}$ is observed for all films at $T=2 \mathrm{~K}$, which is a signature of WAL of surface states. For the 6-nm film, the polarization switching affects the magnitude of $\Delta G$ and sharpness of the cusp appreciably, implying fairly effective of the polarization switching on the surface states. Note that as the film thickness increases the effects of electric-field-induced polarization charges on $\Delta G$ become less pronounced (Fig. 4a), indicating increasing dominance of bulk contributions. With increasing temperature from $T=2 \mathrm{~K}$, the sharp cusp in the conductance is gradually suppressed and almost disappears at $T=50 \mathrm{~K}$, regardless of the polarization states of PMN-PT (Supplementary Figure S10). It has been shown that 2D magnetoconductance associated with the WAL effect can be described by the Hikami-Larkin-Nagaoka (HLN) theory. ${ }^{47,48}$ In the low-field regime, quantum corrections to the conductance are given by

$\Delta G=a \frac{\mathrm{e}^{2}}{2 \pi^{2} \hbar}\left[\psi\left(\frac{1}{2}+\frac{\hbar}{4 e B \ell_{\phi}^{2}}\right)-\ln \left(\frac{\hbar}{4 e B \ell_{\phi}^{2}}\right)\right]$ 


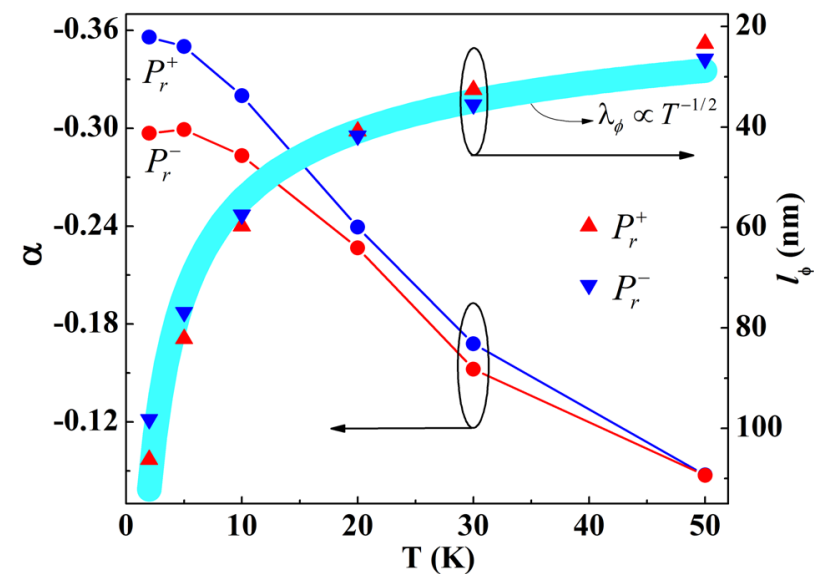

Fig. 5 Temperature dependence of the conductance channel $\alpha$ and the phase coherence length $\ell_{\varphi}$ for the $6-n m$ CBS film when the PMN-PT substrate is in the $P_{r}^{+}$and $P_{r}^{-}$states, respectively. The thick cyan line is the fitting to $\ell_{\varphi}$ using with $p=1$

Here, $\psi$ is the digamma function, $B$ is the magnetic field perpendicular to the film plane, $\ell_{\phi}$ is the phase coherence length with $\ell_{\phi}=\sqrt{D \tau_{\phi}}$, where $D$ and $\tau_{\phi}$ are the electron diffuse constant and phase coherence time, respectively. $\Delta G$ of the $6-\mathrm{nm}$ film at different fixed temperatures for both the $P_{r}^{+}$and $P_{r}^{-}$states (Fig. 4b) can be well fitted by Eq. (3) with the fitting parameters $a$ and $\ell_{\phi}$ shown in Fig. 5, where $a$ at $T=2 \mathrm{~K}$ increases by some $16.7 \%$ (from -0.36 to -0.30 ) as the polarization is switched from $P_{r}^{+}$to $P_{r}^{-}$, indicating a weakening of the conductance channel of the surface states due to the reduction in $n_{2 D}$. With increasing temperature from 2 to $50 \mathrm{~K}$, not only the absolute value of $a$ but also the difference in $a$ between the $P_{r}^{+}$and the $P_{r}^{-}$states $(\Delta a)$, i.e., $\Delta a=\left|a\left(P_{r}^{+}\right)\right|-\left|a\left(P_{r}^{-}\right)\right|$, decreases, indicating both a reduced contribution of the surface states to the conductance and a suppression of the effects of polarization switching on surface states with increasing temperature. Because of enhanced inelastic scattering with increasing temperature, the electron phase coherence length $\ell_{\phi}$ for both polarization states decreases with increasing temperature from 2 to $50 \mathrm{~K}$. The relationship between $\ell_{\phi}$ and temperature $T$ can be rather well described by a power law, $\ell_{\phi} \propto T^{-p / 2}$, with $p=1$ for both polarization states, constituting yet another signature of the surface states in the $6-\mathrm{nm}$ film. ${ }^{20,44}$ One should note that polarization switching from $P_{r}^{+}$to $P_{r}^{-}$has resulted in reducing $\Delta G$ at temperatures $T<20 \mathrm{~K}$ and in its enhancement for $T>20 \mathrm{~K}$ (Fig. 4b). Near the crossover temperature $(T=20 \mathrm{~K})$, the polarization switching has almost no effect on $\Delta G$. According to Eq. (3), this opposite effects of polarization switching on $\Delta G$ above and below $T=20 \mathrm{~K}$ may be related to the opposite trends in $\ell_{\phi}$ induced by polarization switching above and below $T=20 \mathrm{~K}$ (Fig. 5). For $T<20 \mathrm{~K}, \ell_{\phi}$ for the $P_{r}^{+}$state is larger than that for the $P_{r}^{-}$state while for $T>20 \mathrm{~K} \ell_{\phi}$ for the $P_{r}^{+}$state is smaller than that for the $P_{r}^{-}$state. Such opposite changes in $\ell_{\phi}$ are probably related to the polarization switching-induced changes in the inelastic scattering of bulk states, as presented in Tables S1, S2, S3, S4 (Supplementary Materials SII), where the inelastic scattering coefficients $\gamma_{\text {in }}$ are seen to decrease as polarization is switched from $P_{r}^{+}$to $P_{r}^{-}$.

In summary, our work reports the first fabrication of CBS/PMNPT topological ferroelectric field-effect devices and the effects of ferroelectric polarization switching on the electronic properties of CBS films with different thicknesses $(3-80 \mathrm{~nm})$. The results demonstrate that the carrier density, Fermi level, conductance, magnetoconductance, conductance channel, phase coherence length, screening factor of electron-electron interaction of the 6$\mathrm{nm}$ CBS film can be tuned in a reversible and nonvolatile manner by polarization switching at room temperature. Our findings show that the polarization switching has opposite effects on the magnetoconductance and the phase coherence length $\ell_{\phi}$ above and below $T=20 \mathrm{~K}$ for the 6-nm CBS film, suggesting a competition between the surfaces states and the bulk states. The combination of a topological insulator with ferroelectrically and piezoelectrically active PMN-PT would not only provide an alternative effective and convenient approach for tuning the Fermi level and electronic properties of a variety of topological insulators in a reversible and nonvolatile manner, but also may pave a way toward exploring topological insulator/ferroelectric (piezoelectric) hybrid multifunctional devices that can not only take advantage of the ferroelectric field-effect of the structure but also the unique properties of respective materials.

\section{MATERIALS AND METHODS}

PMN-PT single-crystal growth and ferroelectric and piezoelectric measurements

A rhombohedral PMN-PT $(\mathrm{PT}=29 \%)$ single crystal with a size of $\phi 50 \times$ $80 \mathrm{~mm}^{3}$ was grown by the modified Bridgman technique at the Shanghai Institute of Ceramics. The single crystal shows excellent ferroelectric properties $\left(2 P_{r}=76 \mu \mathrm{C} / \mathrm{cm}^{2}\right)$ (Fig. 1e) and an ultra-high piezoelectric coefficient $d_{33} \sim 2500 \mathrm{pC} / \mathrm{N}$. The single crystal was cut into (111)-oriented rectangular plates with a size of $5 \times 5 \times 0.5 \mathrm{~mm}^{3}$ and carefully polished on one side to root-mean-square roughness $R_{\mathrm{q}}<1 \mathrm{~nm}$. Such polished PMN-PT crystals were used as substrates to grow the $\mathrm{Cr}_{x} \mathrm{Bi}_{2-x} \mathrm{Se}_{3}(x=0.11)$ films. Ferroelectric hysteresis loops of PMN-PT substrates were measured using a Precision Multiferroic test system (Radiant Technologies, Inc., USA) at a frequency of $1 \mathrm{~Hz}$ at $T=300 \mathrm{~K}$. The piezoelectric coefficient $d_{33}$ was measured using a $d_{33}$-meter (ZJ-3A, China). Piezoresponse force microscopy (PFM) images of PMN-PT were measured using a Veeco NanoScopeIV Multimode scanning probe microscope.

\section{Thin-film growth}

CBS films were grown on PMN-PT single-crystal substrates by the pulsed laser deposition from a high-purity $\mathrm{CBS}$ target prepared by the spark plasma sintering (SPS) under $50 \mathrm{MPa}$ at $450{ }^{\circ} \mathrm{C}$. The target was ablated by a $\mathrm{KrF}$ excimer laser $(248 \mathrm{~nm})$ with a pulse energy density of $1.2 \mathrm{~J} / \mathrm{cm}^{2}$ and a repetition rate of $1 \mathrm{~Hz}$. The film growth was carried out at a substrate temperature of $350^{\circ} \mathrm{C}$ in $35 \mathrm{~Pa} \mathrm{Ar}$ atmosphere, followed by in situ annealing for $30 \mathrm{~min}$ and then cooled to the room temperature. Film thicknesses were controlled by the number of laser pulses.

\section{Composition and structural characterization}

The phase purity and growth direction of CBS films were characterized by a high-resolution Bruker D8 Discover X-ray diffractometer using $\mathrm{CuK}_{\mathrm{a} 1}$ radiation $(\lambda=1.5406 \AA)$. Transmission electron microscopy (TEM) and energy-dispersive $\mathrm{X}$-ray spectroscopy (EDS) measurements were performed using the Tecnai G2 F20 S-Twin transmission electron microscope. EDS results show that the $\mathrm{Cr}$ doping level is $\sim 11$ at $\%$ and distributes homogeneously in the films (Supplementary Figure S1).

\section{Electronic transport and magnetic property measurements}

The electronic transport properties of CBS films were measured using a Physical Property Measurement System (PPMS-9, Quantum Design), with the direction of the magnetic field perpendicular to the film plane. We particularly note here that, except for the data shown in Fig. If, all the other electronic transport data were obtained by measuring the resistance of the CBS films after the removal of the electric fields so that the effects of electric-field-induced lattice strain due to the electrostriction could be ruled out. Magnetic properties were measured using a SQUID magnetometer (Quantum Design). Using the conducting CBS film and the Ag film $(\sim 100 \mathrm{~nm})$ as top and bottom electrodes, respectively, the poling of PMNPT substrates was achieved by applying a positive or negative dc poling voltage of $330 \mathrm{~V}(\sim 6.6 \mathrm{kV} / \mathrm{cm})$ to the PMN-PT layer along the thickness direction (Supplementary Figure S2). 


\section{DATA AVAILABILITY}

The authors declare that the data supporting the findings of this study are available within the article and its Supplementary Information files.

\section{ACKNOWLEDGEMENTS}

This work was supported by the National Natural Science Foundation of China (Grant Nos. 51572278, 51332007, 51502129, 51872278, and 11574126), the National Basic Research Program of China (Grant Nos. 2016YFA0300103 and 2015CB921201), and the Chinese Academy of Sciences (Grant No. KGZD-EW-T06). H.Z.L. was supported by the National Key R\&D Program (Grant No. 2016YFA0301700) and National Natura Science Foundation of China (Grant No. 11574127). S.N.D. and J.K.F. were supported by National Science Foundation Grant DMR 1400432, USA. Support from Jiangxi Key Laboratory for Two-Dimensional Materials and Jiangxi Engineering Laboratory for Thin Films and Devices is also acknowledged.

\section{AUTHOR CONTRIBUTIONS}

X.W.Z. and J.M.Y. prepared thin films and performed electronic transport measurements. G.Y.G. carried out XRD and TEM measurements. Z.X.X performed magnetic and EDS measurements. M.X. performed ferroelectric and piezoelectric measurements. S.Y.Y and J.X.Z. performed PFM measurements. H.S.L. grew PMN-PT single crystals. X.G.L., W.Y.Z., Y.W., H.Z.L, and J.K.F. participated in the manuscript revision. R. K.Z. and Y.W. conceived the idea. R.K.Z. designed and supervised the research. X.W.Z., S.N.D., Y.K.L. and R.K.Z. wrote the paper.

\section{ADDITIONAL INFORMATION}

Supplementary information accompanies the paper on the npj Quantum Materials website (https://doi.org/10.1038/s41535-018-0125-0).

Competing interests: The authors declare no competing interests.

Publisher's note: Springer Nature remains neutral with regard to jurisdictional claims in published maps and institutional affiliations.

\section{REFERENCES}

1. Ando, Y. Topological insulator materials. J. Phys. Soc. Jpn 82, 102001 (2013).

2. Shen, J. Y. et al. Nematic topological superconducting phase in $\mathrm{Nb}$-doped $\mathrm{Bi}_{2} \mathrm{Se}_{3}$. npj Quantum Mater. 2, 59 (2017).

3. Taskin, A. A. et al. Planar Hall effect from the surface of topological insulators. Nat. Commun. 8, 1340 (2017).

4. Wang, Z. Y. et al. Tuning carrier type and density in $\mathrm{Bi}_{2} \mathrm{Se}_{3}$ by Ca-doping. Appl. Phys. Lett. 97, 042112 (2010).

5. Kuroda, K. et al. Hexagonally deformed Fermi surface of the 3D topological insulator $\mathrm{Bi}_{2} \mathrm{Se}_{3}$. Phys. Rev. Lett. 105, 076802 (2010).

6. Kim, Y. S. et al. Thickness-dependent bulk properties and weak antilocalization effect in topological insulator $\mathrm{Bi}_{2} \mathrm{Se}_{3}$. Phys. Rev. B 84, 073109 (2011)

7. Feng, $X$. et al. Thickness dependence of the quantum anomalous Hall effect in magnetic topological insulator films. Adv. Mater. 28, 6386-6390 (2016).

8. Park, S. H. et al. Reversible Fermi level tuning of a $\mathrm{Sb}_{2} \mathrm{Te}_{3}$ topological insulator by structural deformation. Nano Lett. 15, 3820-3826 (2015).

9. Qi, S. F. et al. Strain-modulated ferromagnetism and band gap of $\mathrm{Mn}$ doped $\mathrm{Bi}_{2} \mathrm{Se}_{3}$. Sci. Rep. 6, 29161 (2016).

10. Liu, Y. et al. Tuning Dirac states by strain in the topological insulator $\mathrm{Bi}_{2} \mathrm{Se}_{3}$. Nat. Phys. 10, 294-299 (2014).

11. Liu, W. L. et al. Anisotropic interactions and strain-induced topological phase transition in $\mathrm{Sb}_{2} \mathrm{Se}_{3}$ and $\mathrm{Bi}_{2} \mathrm{Se}_{3}$. Phys. Rev. B 84, 245105 (2011).

12. Storz, O. et al. Mapping the effect of defect-induced strain disorder on the Dirac states of topological insulators. Phys. Rev. B 94, 121301(R) (2016).

13. Bera, A., Pal, K., Muthu, D. V. S., Waghmare, U. V. \& Sood, A. K. Pressure-induced phase transition in $\mathrm{Bi}_{2} \mathrm{Se}_{3}$ at $3 \mathrm{GPa}$ : electronic topological transition or not? J. Phys.: Conden. Mater. 28, 105401 (2016).

14. Liu, W. Q. et al. Enhancing magnetic ordering in $\mathrm{Cr}$-doped $\mathrm{Bi}_{2} \mathrm{Se}_{3}$ using high- $T_{C}$ ferrimagnetic insulator. Nano Lett. 15, 764-769 (2015).

15. Zheng, G. L. et al. Weak localization effect in topological insulator micro flakes grown on insulating ferrimagnet $\mathrm{BaFe}_{12} \mathrm{O}_{19}$. Sci. Rep. 6, 21334 (2016).

16. Chang, C.-Z. et al. Simultaneous electrical-field-effect modulation of both top and bottom Dirac surface states of epitaxial thin films of three dimentional topological insulators. Nano Lett. 15, 1090-1094 (2015).
17. Steinberg, H., Lalöe, J.-B., Fatemi, V., Moodera, J. S. \& Jarillo-Herrero, P. Electrically tunable surface-to-bulk coherent coupling in topological insulator thin films. Phys. Rev. B 84, 233101 (2011).

18. Xu, Y., Miotkowski, I. \& Chen, Y. P. Quantum transport of two-species Dirac fermions in dual-gated three-dimensional topological insulators. Nat. Commun. 7 11434 (2016)

19. Fan., Y. et al. Dual-gated topological insulator thin-film device for efficient Fermilevel tuning. ACS Nano 9, 4050-4055 (2015).

20. Tian, J. F. et al. Quantum and classical magnetoresistance in ambipolar topological insulator transistors with gate-tunable bulk and surface conduction. Sci. Rep. 4, 4859 (2014).

21. Chang, C.-Z. et al. Experimental observation of the quantum anomalous Hall effect in a magnetic topological insulator. Science 340, 167-170 (2013).

22. Chen, J. et al. Tunable surface conductivity in $\mathrm{B}_{\mathrm{i}} 2 \mathrm{~S}_{\mathrm{e}} 3$ revealed in diffusive electron transport. Phys. Rev. B 83, 241304(R) (2011).

23. Checkelsky, J. G., Ye, J. T., Onose, Y., Iwasa, Y. \& Tokura, Y. Dirac-fermion-mediated ferromagnetism in a topological insulator. Nat. Phys. 8, 729-733 (2012).

24. Nelson, J. J. \& Goldman, A. M. Metallic state of low-mobility silicon at high carrier density induced by an ionic liquid. Phys. Rev. B 91, 241304(R) (2015).

25. Kim, D. et al. Surface conduction of topological Dirac electrons in bulk insulating $\mathrm{Bi}_{2} \mathrm{Se}_{3}$. Nat. Phys. 8, 459-463 (2012).

26. Liao, J. et al. Observation of Anderson localization in ultrathin films of threedimensional topological insulators. Phys. Rev. Lett. 114, 216601 (2015).

27. Dai, J. X. et al. Toward the intrinsic limit of the topological insulator $\mathrm{Bi}_{2} \mathrm{Se}_{3}$. Phys. Rev. Lett. 117, 106401 (2016).

28. Wang, Y. et al. Gate-controlled surface conduction in Na-doped $\mathrm{Bi}_{2} \mathrm{Te}_{3}$ topological insulator nanoplates. Nano Lett. 12, 1170-1175 (2012).

29. Lei, B. et al. Evolution of high-temperature superconductivity from a low- $T_{c}$ phase tuned by carrier concentration in FeSe thin flakes. Phys. Rev. Lett. 116, 077002 (2016).

30. $\mathrm{Yu}, \mathrm{Y}$. J. et al. Gate-tunable phase transitions in thin flakes of $1 \mathrm{~T}-\mathrm{TaS}_{2}$. Nat Nanotechnol. 10, 270-276 (2015).

31. Leng, $\mathrm{X}$. et al. Insulator to metal transition in $\mathrm{WO}_{3}$ induced by electrolyte gating npj Quantum Mater. 2, 35 (2017).

32. Li, Y. F. et al. Electrostatic tuning of Kondo effect in a rare-earth-doped wideband-gap oxide. Phys. Rev. B 87, 155151 (2013).

33. Lu, N. P. et al. Electric-field control of tri-state phase transformation with a selective dual-ion switch. Nature 546, 124 (2017).

34. Liu, M. H. et al. Electron interaction-driven insulating ground state in $\mathrm{Bi}_{2} \mathrm{Se}_{3}$ topological insulators in the two-dimensional limit. Phys. Rev. B 83, 165440 (2011).

35. Zhu, Q. X. et al. Ultrahigh tunability of room temperature electronic transport and ferromagnetism in dilute magnetic semiconductor and PMN-PT single-crystalbased field effect transistors via electric charge mediation. Adv. Funct. Mater. 25 1111-1119 (2015).

36. Chen, L. et al. Semiconductor/piezoelectrics hybrid heterostructures with highly effective gate-tunable electrotransport and magnetic behaviors. ACS Appl. Mater. Interfaces 8, 26932-26937 (2016).

37. Analytis, J. G. et al. Two-dimensional surface state in the quantum limit of a topological insulator. Nat. Phys. 6, 960-964 (2010).

38. Choi, Y. H. et al. Simple tuning of carrier type in topological insulator $\mathrm{Bi}_{2} \mathrm{Se}_{3}$ by $\mathrm{Mn}$ doping. Appl. Phys. Lett. 101, 152103 (2012).

39. Okada, K. N. et al. Enhanced photogalvanic current in topological insulators via Fermi energy tuning. Phys. Rev. B 93, 081403(R) (2016).

40. Brahlek, M., Koirala, N., Bansal, N. \& Oh, S. Transport properties of topological insulators: band bending, bulk metal-to-insulator transition, and weak antilocalization. Solid State Commun. 215-216, 54-62 (2015).

41. Lu, H.-Z. \& Shen, S.-Q. Finite-temperature conductivity and magnetoconductivity of topological insulators. Phys. Rev. Lett. 112, 146601 (2014).

42. Haazen, P. P. J. et al. Ferromagnetism in thin-film $\mathrm{Cr}$-doped topological insulator $\mathrm{Bi}_{2} \mathrm{Se}_{3}$. Appl. Phys. Lett. 100, 082404 (2012).

43. Zhang, Z. C. et al. Magnetic quantum phase transition in $\mathrm{Cr}$-doped $\mathrm{B}_{\mathrm{i}} 2\left(\mathrm{Se}_{x} \mathrm{Te} \mathrm{e}_{1-x)} 3\right.$ driven by the Stark effect. Nat. Nanotechnol. 12, 953 (2017).

44. Kuntsevich, A. Y. et al. Low-temperature Hall effect in bismuth chalcogenides thin films. Phys. Rev. B 94, 235401 (2016)

45. Wang, J. et al. Evidence for electron-electron interaction in topological insulator thin film. Phys. Rev. B 83, 245843 (2011).

46. Checkelsky, J. G. et al. Quantum interference in macroscopic crystals of nonmetallic $\mathrm{Bi}_{2} \mathrm{Se}_{3}$. Phys. Rev. Lett. 103, 246601 (2009).

47. Hikami, S., Larkin, A. I. \& Nagaoka, Y. Spin-orbit interaction and magnetoresistance in the two dimensional random system. Prog. Theor. Phys. 63, 707-710 (1980).

48. Liu, M. H. et al. Crossover between weak antilocalization and weak localization in a magnetically doped topological insulator. Phys. Rev. Lett. 108, 036805 (2012). 
Access This article is licensed under a Creative Commons Attribution 4.0 International License, which permits use, sharing, adaptation, distribution and reproduction in any medium or format, as long as you give appropriate credit to the original author(s) and the source, provide a link to the Creative Commons license, and indicate if changes were made. The images or other third party material in this article are included in the article's Creative Commons license, unless indicated otherwise in a credit line to the material. If material is not included in the article's Creative Commons license and your intended use is not permitted by statutory regulation or exceeds the permitted use, you will need to obtain permission directly from the copyright holder. To view a copy of this license, visit http://creativecommons. org/licenses/by/4.0/.

(c) The Author(s) 2018 